ARTICLE

Received 3 Oct 2012 | Accepted 18 Jan 2013 | Published 26 Feb 2013 DOl: 10.1038/ncomms2514

\title{
Translation of HTT mRNA with expanded CAG repeats is regulated by the MID1-PP2A protein complex
}

Sybille Krauß ${ }^{1}$, Nadine Griesche ${ }^{1}$, Ewa Jastrzebska ${ }^{2,3}$, Changwei Chen ${ }^{4}$, Désiree Rutschow ${ }^{4}$, Clemens Achmüller ${ }^{5,6, \dagger}$, Stephanie Dorn', Sylvia M. Boesch ${ }^{6}$, Maciej Lalowski ${ }^{7,8}$, Erich Wanker ${ }^{7}$, Rainer Schneider ${ }^{3,5} \&$ Susann Schweiger $3,4,9$

Expansion of CAG repeats is a common feature of various neurodegenerative disorders, including Huntington's disease. Here we show that expanded CAG repeats bind to a translation regulatory protein complex containing MID1, protein phosphatase $2 \mathrm{~A}$ and $40 \mathrm{~S}$ ribosomal $\mathrm{S} 6$ kinase. Binding of the MID1-protein phosphatase 2A protein complex increases with CAG repeat size and stimulates translation of the CAG repeat expansion containing messenger RNA in a MID1-, protein phosphatase 2A- and mammalian target of rapamycindependent manner. Our data indicate that pathological CAG repeat expansions upregulate protein translation leading to an overproduction of aberrant protein and suggest that the MID1-complex may serve as a therapeutic target for the treatment of CAG repeat expansion disorders.

\footnotetext{
${ }^{1}$ German Center for Neurodegenerative Diseases (DZNE), Sigmund-Freud-Str. 25, 53127, Bonn, Germany. ${ }^{2}$ Department of Dermatology, Charité University Hospital, Charitéplatz1, 10117 Berlin, Germany. ${ }^{3}$ Department of Human Molecular Genetics (Ropers), Max Planck Institute for Molecular Genetics, Ihnestrasse 73, 14195 Berlin, Germany. ${ }^{4}$ Division of Neuroscience, Medical Research Institute, Ninewels Hospital \& Medical School, Dundee DD19SY, UK. ${ }^{5}$ Institute of Biochemistry and Center for Molecular Biosciences Innsbruck (CMBI), Innrain 80/82, A-6020 Innsbruck, Austria. ${ }^{6}$ Department of Neurology, Innsbruck Medical University, Anichstrasse 35, 6020 Innsbruck, Austria. ${ }^{7}$ Proteomics and Molecular Mechanisms of Neurodegenerative Diseases, Max Delbrück Center for Molecular Medicine (MDC) Berlin-Buch, Robert-Rössle-Strasse 10, 13092 Berlin, Germany. ${ }^{8}$ Biomedicum Helsinki, Meilahti Clinical Proteomics Core Facility and Folkhälsan Research Center, University of Helsinki, PO Box 63, (Haartmaninkatu 8), Helsinki Fl-00014, Finland. ${ }^{9}$ Institute for Human Genetics, Medical School, University of Mainz, 55131 Mainz, Germany. † Present address: Sandoz GmbH, Biochemiestrasse 10, 6250 Kundl, Austria. Correspondence and requests for materials should be addressed to S.K. (email: Sybille.Krauss@dzne.de) or S.S. (email: susann.schweiger@unimedizin-mainz.de).
} 
untington's disease (HD) (OMIM accession code 143100) is caused by an expanded CAG repeat in exon 1 of the Huntingtin (HTT) gene. HD is characterized by progressive neuronal cell death associated with choreatic movements, as well as dementia. The disease progresses over time and is invariably fatal. In most cases onset occurs in mid-life (35-50 years). Age at onset is inversely correlated to the length of the extensions of the CAG stretches (reviewed in Gil and Rego $^{1}$ ).

A pathological hallmark of several CAG repeat expansion disorders is the formation of protein aggregates in neurons. Expanded CAG sequences are translated into elongated polyglutamine (polyQ) stretches. This renders the respective proteins insoluble and leads to the formation of protein aggregates ${ }^{2-7}$.

In some CAG repeat expansion disorders gain-of-function at the RNA instead of the protein level has been proposed ${ }^{8}$. CAG sequences form double-stranded RNA structures, the stability of which increases with repeat-numbers ${ }^{9-11}$. For example the CAG repeat region of the HTT messenger RNA (mRNA) forms double-stranded hairpin structures ${ }^{12}$. Some proteins bind to such CAG structures in a repeat size-dependent manner ${ }^{13}$. Thus, it is thought that expansion of CAG repeat structures leads to the formation of pathological RNA-protein complexes, which cause damage in both-the nucleus where they influence the splicing machinery-and in the cytosol where they interfere with mRNA processing and translation ${ }^{12,14}$.

Several processes in gene expression, from mRNA transcription and stability to translation, involve RNA-binding proteins, which assemble with the mRNAs into mRNP (messenger ribonucleoprotein) complexes ${ }^{15}$. One of such mRNP complexes contains the MID1 protein, the catalytic subunit of protein phosphatase 2A (PP2Ac) and 40S ribosomal S6 kinase (S6K), a target of mammalian target of rapamycin (mTOR) kinase and PP2 $\mathrm{A}^{16,17}$. MID1 is a ubiquitin ligase that binds the $\alpha 4$ protein, a regulatory subunit of $\mathrm{PP} 2 \mathrm{~A}$. Through this interaction MID1 ubiquitinates PP2Ac and targets it towards ubiquitin-specific degradation by the proteasome. Therefore, MID1 is a negative regulator of $\mathrm{PP} 2 \mathrm{Ac}^{18}$. Through this negative regulatory influence on PP2A activity, MID1 also controls the activity of the mTOR kinase. High levels of PP2A activity in MID1-deficient cells abolish the interaction between $\mathrm{mTOR}$ and raptor, which is an essential cofactor of mTOR activity ${ }^{19}$. Accordingly, a significant reduction of $\mathrm{mTOR}$ activity is found in these cells.

mTOR and its counteracting phosphatase PP2A have important roles in the regulation of protein translation. Both enzymes control the phosphorylation and thereby the activity of several translation regulators. For example, mTOR phosphorylates and activates $\mathrm{S} 6 \mathrm{~K}$ and $4 \mathrm{E}-\mathrm{BP} 1$, both of which participate in the assembly of the eukaryotic translation initiation factor (elF)3 complex and the initiation of translation: when inactive, S6K associates with the elF3 preinitiation complex. Upon stimulation mTOR localizes to the elF3 complex and activates S6K via phosphorylation $^{20,21}$. Activated S6K phosphorylates and enhances the activity of its targets elF4B and S6 (ref. 21). The $5^{\prime}$-untranslated region (UTR) of specific mRNAs folds into stable secondary structures, which principally suppress protein translation ${ }^{22,23}$. Activated elF4B unwinds and linearizes such UTRs, allowing initial binding of the $40 \mathrm{~S}$ ribosome and scanning towards the translation initiation codon ${ }^{24,25}$. Concurrently mTOR phosphorylates $4 \mathrm{E}-\mathrm{BP} 1$, an inhibitory regulator of elF4E, leading to dissociation of 4E-BP1 from the cap complex, thereby allowing binding of elF4E to elF4G and assembly of the preinitiation complex finally resulting in translation initiation ${ }^{21,25}$. mTOR dependent phosphorylation of $\mathrm{S} 6 \mathrm{~K}$ and $4 \mathrm{E}-\mathrm{BP} 1$, therefore, efficiently supports protein translation, while their dephosphorylation by PP2A inhibits it.
Besides regulating $\mathrm{PP} 2 \mathrm{~A}$ and $\mathrm{mTOR}$ activities, the MID1complex contains polyribosomes and several translation factors and is able to bind mRNA thereby forming a microtubuleassociated mRNP complex ${ }^{17}$.

As we have shown previously MID1 and $\alpha 4$ stimulate translation of mRNAs that are associated with the MID1 protein complex $^{16}$. Here we demonstrate that the MID1 protein together with its interaction partners PP2A and S6K binds mutant HTT mRNA via its elongated CAG repeats. This interaction results in enhanced translation of mutant HTT mRNA, an effect that can be significantly reduced by knockdown of MID1. The PP2A inhibitor okadaic acid (OA) can block this reduction in translation caused by MID1 knockdown. Furthermore, the mTOR inhibitor temsirolimus can block the increased translation of HTT mRNA with expanded CAG repeats. Taken together our data indicate that expanded CAG repeats in mRNAs trigger translation in a MID1/PP2A/mTOR dependent manner and they suggest that the MID1-complex might be a novel, promising drug target for the development of therapeutic strategies against CAG repeat expansion disorders like HD.

\section{Results}

MID1-complex binds CAG repeats in a length dependent manner. CAG repeat RNAs fold into secondary hairpin structures $^{9-12}$. To give an idea of how these hairpins could look like, we performed in silico RNA-structure predictions of CAG repeat stretches with different repeat sizes. A CAG-containing loop that elongates with increasing CAG number is particularly pronounced (Supplementary Fig. S1). The MID1 protein complex binds mRNAs through G-rich RNA motifs forming stable secondary structures ${ }^{16,17,26}$. Thus, CAG repeat stretches, which are G-rich structures and form stable hairpins, could be recognized by the MID1-complex. To test this, we performed RNA-protein pull-down assays. Equal amounts of in vitro transcribed, biotinylated HTT-exon1-mRNA carrying either 20 (representing physiological numbers of repeats) or 51 (representing pathological numbers of repeats) CAG repeats were incubated with $\mathrm{HeLa}$ cell extracts that either contained FLAG-MID1 (Fig. 1a) or no recombinant protein (Fig. 1b). RNAs were purified with streptavidin-coated beads and bound proteins were analysed by western blotting using (i) an anti-FLAG antibody (Fig. 1a), (ii) an antibody detecting endogenous MID1 (Fig. 1b) and (iii) an antibody detecting glyceraldehyde 3-phosphate dehydrogenase as a negative control that does not bind to the MID1 protein complex. In addition, an experiment without RNA was performed. Although binding of MID1 to the 20 CAG, repeat RNA was weak, a strong band of the MID1 protein (FLAG-tagged and endogenous) was detected in the sample with the 51 repeat RNA. Glyceraldehyde 3-phosphate dehydrogenase did not bind to any of the RNAs.

Furthermore, the MID1 interacting proteins of the PP2A/ mTOR signalling cascade, PP2Ac and S6K, also bound to RNA containing 51 CAG repeats, while no or only very weak binding was observed with RNA containing 20 CAG repeats (Fig. 1c).

MID1 dependency of the binding of S6K and PP2Ac to HTT mRNAs with expanded CAG repeats was tested in an RNAse assisted chromatography ${ }^{27}$ in cells with and without MID1 knockdown. Equal amounts of in vitro transcribed HTT-exon1mRNA with either 20 or 51 CAG repeats were incubated with cell lysates that were either transfected with non-silencing control or MID1-specific short interfering RNA (siRNA) oligonucleotides. RNAs were immobilized on magnetic beads and RNA-bound proteins were eluted from the beads using RNAse. RNA-bound proteins were analysed by western blotting using antibodies detecting MID1, S6K and PP2Ac. As a negative control an 
a

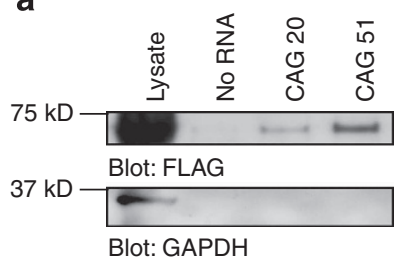

b

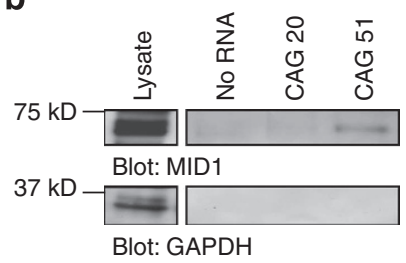

C

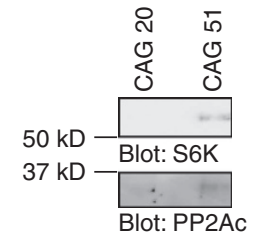

d

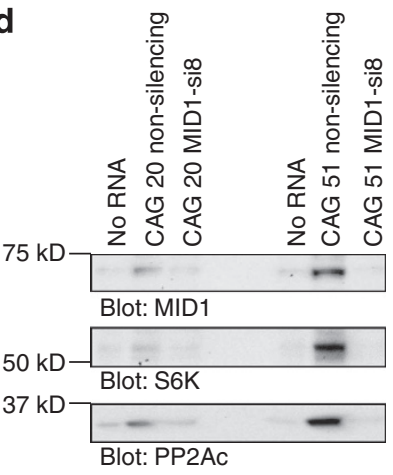

Figure 1 | The MID1-complex binds CAG-repeat-motifs in a size-

dependent manner. (a) RNA-protein pull-down of FLAG-MID1 with RNA containing 20 or 51 CAG repeats (CAG 20, CAG 51) or without RNA (no RNA). RNA-bound proteins were analysed on western blots detecting FLAG-MID1. Glyceraldehyde 3-phosphate dehydrogenase (GAPDH) was detected as negative control that does not bind to the MID1 protein complex. (b) RNA-protein pull-down of endogenous MID1 as in (a). (c) RNA-protein pull-down assay as described in (a). RNA-bound proteins were analysed on a western blot using specific antibodies detecting PP2Ac and S6K. (d) RNAse assisted chromatography of protein lysates from cells transfected with non-silencing control or MID1-specific siRNAs. Proteins bound to RNA containing 20 or 51 CAG repeats were analysed on a western blot using specific antibodies detecting MID1, PP2Ac and S6K. An experiment without RNA (no RNA) was included as negative control.

experiment without RNA was performed. Although binding of MID1, S6K, and PP2Ac to the 20 CAG repeat RNA was weak, strong bands of the three proteins were detected in the sample with the 51 repeat RNA. In the MID1 knockdown samples neither MID1 nor S6K or PP2Ac could be detected in the elution fraction suggesting that MID1 mediates binding of S6K and PP2Ac to the CAG repeat containing RNA (Fig. 1d, for knockdown control see Supplementary Fig. S2).

The preceding data suggest that longer CAG repeats bind the MID1 protein complex more efficiently than shorter. In order to verify this, we repeated the RNA-protein pull-down experiment using HeLa cells overexpressing MID1-FLAG with increasing salt concentrations. At $100 \mathrm{mM}$ salt both RNAs, with 20 and 51 CAG repeats bound MID1 and PP2Ac. At 150 and $200 \mathrm{mM}$ the 20 CAG repeat containing RNA lost MID1 and PP2Ac-binding capabilities. By contrast, significant amounts of MID1 and PP2AC were still bound to the CAG 51 containing RNA (Supplementary Fig. S3) suggesting that the binding affinity of the MID1 protein complex increases with the length of the repeat.

HTT translation increases with the number of CAG repeats. The experiments described above, and the role of the MID1 protein complex in the regulation of translation efficiency of mRNAs specifically attached to it $^{16}$ made us hypothesize that (i) the MID1-complex could control the translation of mRNAs with expanded CAG repeats, and (ii) the amount of protein that is synthesized from a CAG repeat containing mRNA increases with the length of the repeat.

To examine whether the repeat size influences the amount of translated protein, mouse embryonic fibroblasts (Atg $+/+)$ were transfected with expression-vectors producing an N-terminal HTT-fragment covering the first 500 amino acids (HTT-500AA) with either 17 or 49 glutamines (Q) together with a green fluorescent protein (GFP)-expression vector as an internal transfection control. Cell extracts were analysed by western blotting. For quantification HTT-500AA signals were related to GFP and Q17 expression was set to 1 . A significantly higher production of protein with 49 than with 17 Q-repeats was revealed (Fig. 2a).

Autophagy has previously been shown to have an important role in the clearance of protein with polyQ stretches ${ }^{28}$. To exclude that unbalanced autophageal degradation causes the seen effect, the above-mentioned experiments were repeated in autophagy negative mouse embryonic fibroblasts, which were derived from mice deficient for Atg5 (Atg $-/-)^{29}$. While expression of the HTT-500AA-Q49 protein was still significantly higher than of the Q17 protein, the difference between the Q49 and the Q17 protein was smaller in the Atg $-I-$ cells than in the Atg $+/+$ cells (Fig. 2a). The observed differences in protein expression between both cell lines could be partially caused by autophageal imbalances that favour protein with shorter Q-stretches. The difference seen between the Q49 and Q17 in the Atg - / - , however, is caused by an autophagy independent mechanism. No differences in levels of the mRNAs of the Q49 and Q17 constructs were detected in both cell lines (Supplementary Fig. S4) suggesting that differences in the translation efficiency underlie the seen effects (Fig. 2a).

In a complementary set of experiments, low and high-number CAG repeats were cloned into the $3^{\prime}$-UTR of a gene encoding firefly luciferase. These reporter constructs permitted the analysis of effects mediated by CAG repeats in the mRNA without being translated into polyQ stretches. Three different constructs (empty vector: pGL3, pGL3-CAG14 and pGL3-CAG47) were subjected to an in vitro translation assay, where equal amounts of in vitro transcribed RNA from the luciferase constructs were in vitro translated in rabbit reticulocyte extracts (schematic of assay details see Supplementary Fig. S5). No difference in luciferase activities between the empty vector and the construct carrying 14 CAG repeats, but a significant increase in firefly activity in the sample with 47 CAG repeats was observed (Fig. 2b), indicating that the mRNA with a pathogenic CAG repeat is translated more efficiently than the mRNA with a non-pathogenic CAG sequence.

This finding could be confirmed in U373MG-cells, a glial cell line isolated from an astrocytoma, which were transfected with the previously described luciferase constructs using a dual-luciferase reporter assay. This system is based on the co-transfection of a firefly luciferase construct with a renilla luciferase construct to control for transfection efficiency. Although there was no significant difference between the empty vector and the construct carrying 14 CAG repeats, a clear increase in firefly luciferase activity was seen with the 47 CAG repeat construct (Fig. 2c). By contrast, no difference between the different constructs on the mRNA level was observed (Fig. 2d). This provides evidence that increased firefly luciferase activity of the 47 CAG repeat construct was due to increased translation. Similar results were obtained in HeLa cells (Supplementary Fig. S6a,b).

While also coding for glutamine on the protein levels, CAA codons do not form hairpin structures and have been shown to interrupt secondary structures formed by CAG repeats ${ }^{9,30}$. In order to test if the hairpin structure is necessary for the seen effect on luciferase activity, luciferase reporter assays using constructs in which the CAG repeats are interrupted by CAA triplets (for exact sequence see Supplementary Table S1, structural predictions see Supplementary Fig. S7a) have been performed. Clearly, insertion of 
a

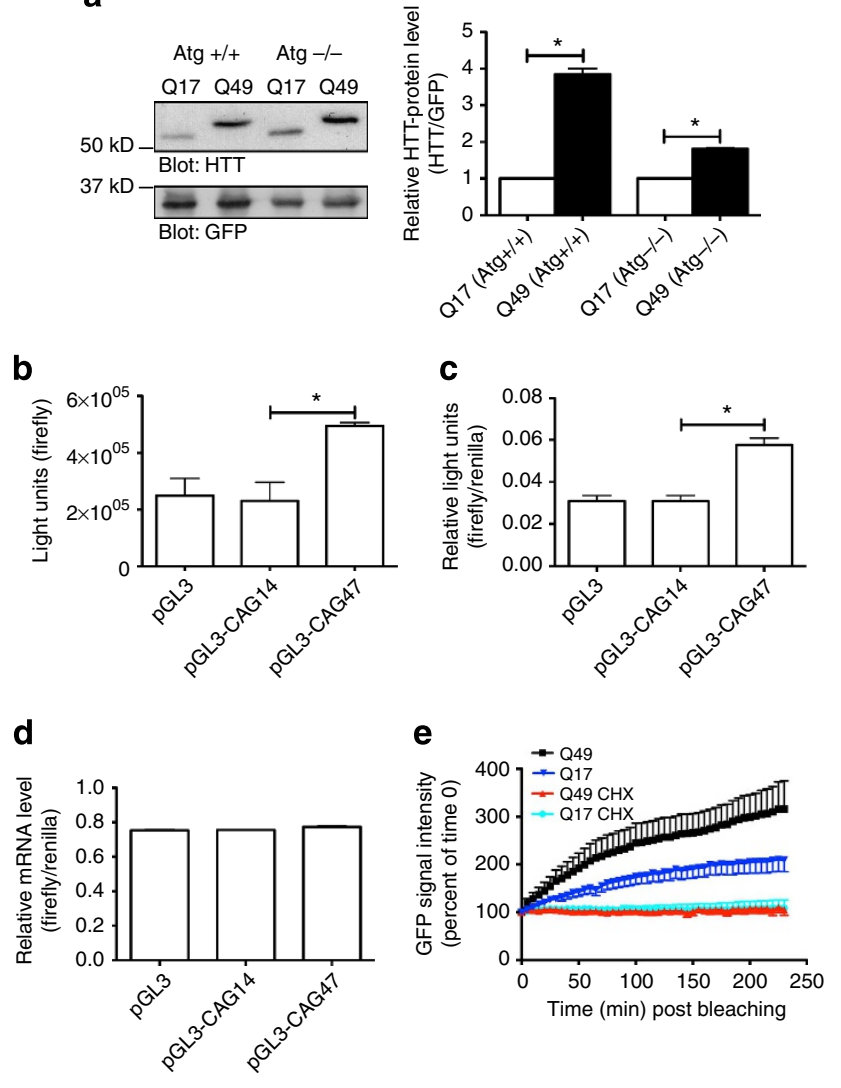

Figure 2 | Protein synthesis increases with repeat size in vitro.

(a) Expression of the first 500 amino acids of the human Huntingtin protein carrying either 17 (HTT-500AA-Q17) or 49 glutamines (HTT-500AA-Q49) in autophagy positive (Atg $+/+$ ) and autophagy negative (Atg $-/-$ ) cells is shown. Left: western blot analysis of Atg $+/+$ and Atg $-/-$ cells co-transfected with pEGFP-C1 and HTT-500AA-Q17/HTT-500AA-Q49.

Right: quantifications of relative HTT-500AA protein normalized to GFP are shown, signals for Q17 were set to 1. Samples were measured in triplicates. Mean values \pm s.d. are shown. $P<0.001$. (b) In vitro translation assay of firefly luciferase carrying no, 14, or 47 CAG repeats in its $3^{\prime}-U T R$ ( $p G L 3$, pGL3-CAG14, pGL3-CAG47). Firefly activity is shown. Samples were measured in triplicates. Mean values \pm s.d. are shown. $P<0.000002$. (c) Dual-luciferase assay of U373MG-cells transfected with constructs used in $\mathbf{b}$ is shown. Columns represent light units of firefly luciferase normalized to renilla. Samples were measured in triplicates. Mean values \pm s.d. are shown. $P<0.0004$. (d) Real-time PCR analysis of cells from c measuring firefly luciferase-mRNA expression normalized to renilla mRNA expression is shown. Samples were measured in quadruplicates. Mean values \pm s.d. are shown. (e) FRAP-based assay showing GFP-HTT-exon1 translation in living cells. HeLa cells were transfected with GFP-HTT-exon1 constructs, either containing 17 CAG (Q17, $n=32)$ or 49 CAG repeats ( $Q 49, n=16$ ). Twelve hours after transfection cells were bleached and recovery of green fluorescence was measured. Also, analysis of the GFP-HTT-exon1 Q17 $(n=28)$ and Q49 $(n=27)$ constructs with cycloheximide $(\mathrm{CHX})$ is shown. GFP-signal recovery over time is shown. Lines represent mean values of all cells analysed. Data are presented as mean values \pm s.e.m. Q17 versus Q49 $P<0.0001$, Q49 versus Q49 + CHX $P<0.0001$

CAA interruptions into the CAG repeat led to a significant reduction in luciferase signal (Supplementary Fig. S7b).

Additionally, we developed a novel live-cell imaging assay based on FRAP (fluorescence recovery after photobleaching) to monitor translation. In contrast to standard FRAP applications, which are used to monitor diffusion/transport of fluorescently labelled
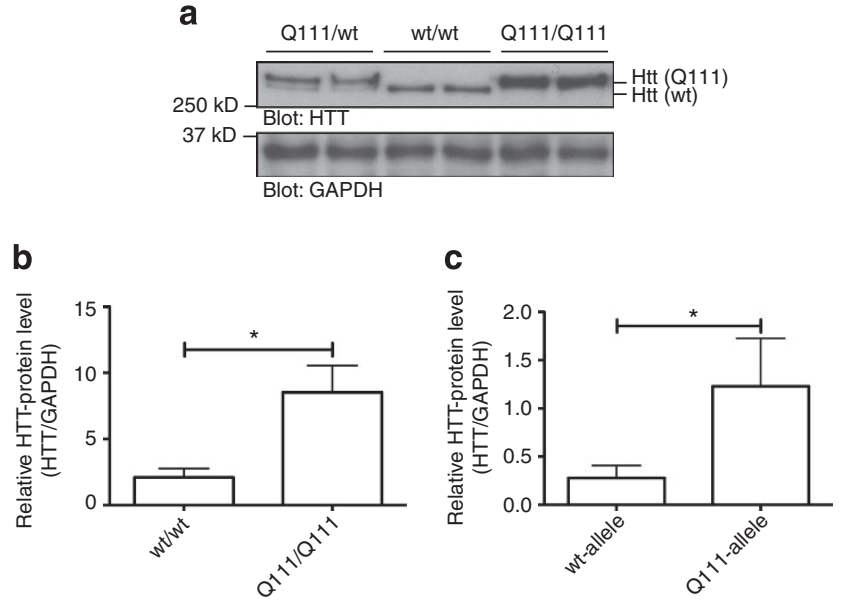

Figure 3 | Protein level increases with repeat size in vivo. (a) Western blot analysis of brain extracts from wild-type (wt), heterozygous (Q111/wt) and homozygous (Q111/Q111) knock-in mice carrying 111 CAG repeats in exon 1 of HTT is shown. Two HTT bands were detected, one smaller translated from the wild-type, one bigger translated from the mutant allele. Glyceraldehyde 3-phosphate dehydrogenase (GAPDH) was analysed as control. (b) Quantifications of bands from homozygous and wild-type animals from a are shown. (c) Quantifications of bands from heterozygous animals (protein expressed from the wild-type compared with protein expressed from the mutant allele) from $\mathbf{a}$ are shown. Three animals of each genotype were quantified. Mean values \pm s.d. are shown. $P<0.007$.

proteins after bleaching of a defined area in the cell, we used FRAP in a whole-cell experimental set-up. Reporter constructs carrying HTT exon1 with 17 or 49 CAGs fused to GFP were transfected into HeLa cells. Twelve hours after transfection the entire cell was bleached and recovery of the fluorescent signal was monitored over $4 \mathrm{~h}$. By bleaching the entire cell, we prohibited signal recovery due to diffusion of fluorescently labelled proteins into the imaging area. Therefore, only newly synthesized GFPprotein could be imaged. Clearly, GFP synthesized from a Q49 construct recovered significantly faster than from the Q17 construct (Fig. 2e, Supplementary Movie 1 and 2, Supplementary Fig. S8). Neither differences on the mRNA levels (Supplementary Fig. S9a) nor different transfection efficiencies of the two constructs were observed (Supplementary Fig. S9b).

To further verify, that the fluorescence recovery was caused by newly synthesized protein, we performed the same experiment with the translation inhibitor cycloheximide. As shown in Fig. 2e (and Supplementary Movie 3), cycloheximide completely blocked the GFP-signal recovery.

To analyse if an expanded CAG repeat stretch leads to an increase in protein production also in vivo, a knock-in mouse model for $\mathrm{HD}$ (B6.129- $\mathrm{Htt}^{\mathrm{tm} 5 \mathrm{Mem}} / \mathrm{J}$, Jackson's Lab stock no.003598), which carries 111 CAG repeats in exon 1 of the endogenous murine HTT-gene was used. Brain lysates from 2-months-old mice (wild-type, heterozygous and homozygous mutation carrier) were analysed on western blots using an HTTantibody detecting the region $\mathrm{N}$-terminal of the CAG repeat. A smaller band corresponding to wild-type HTT protein and a bigger band corresponding to mutant HTT protein, were detected. Significantly more mutant than wild-type protein was detected suggesting that the translation rate of RNA from the mutated allele is higher than from the wild-type allele in heterozygous and homozygous animals (Fig. 3a, b). Together with previous reports showing that mRNA levels of mutant HTT decrease with increasing repeat lengths in $\mathrm{HD}$ mouse models ${ }^{31,32}$, these data 
a

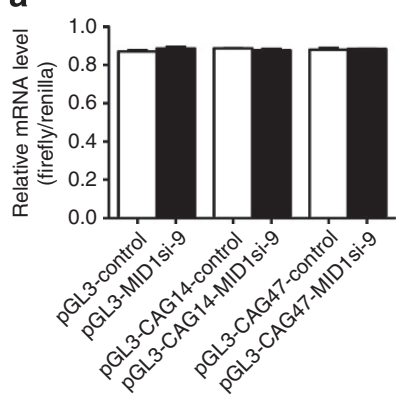

b

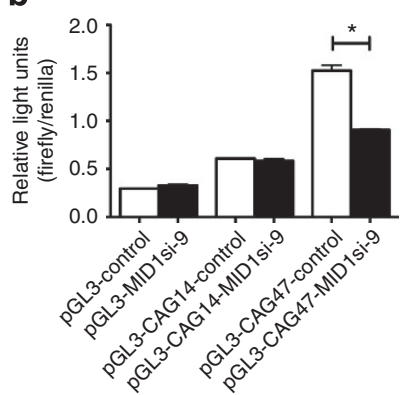

C

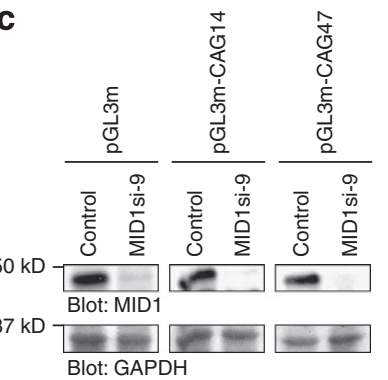

Figure 4 | The MID1-complex regulates translation of mRNAs with expanded CAG repeats. Dual-luciferase assays of HeLa cells are shown expressing the firefly luciferase constructs from Fig. 2 co-transfected with either non-silencing (pGL3-control, pGL3-CAG14-control, pGL3-CAG47control) or MID1-specific siRNA oligonucleotides (pGL3-MID1si-9, pGL3CAG14-MID1si-9, pGL3-CAG47-MID1si-9). (a) Real-time PCR analysis showing relative of firefly to renilla mRNA expression. Samples were measured in quadruplicates. (b) Cells from a were analysed for luciferase activity. Columns represent relative light units (firefly/renilla). Samples were measured in triplicates. Mean values \pm s.d. are shown. $P<0.001$. (c) Western blots for knockdown control of cells from a detected with MID1-specific and glyceraldehyde 3-phosphate dehydrogenase (GAPDH)-specific antibodies are shown.

support the hypothesis that an expanded CAG repeat increases the translation efficiency of mutant HTT mRNA in vivo.

MID1 regulates translation of CAG repeat mRNAs. To assess the importance of the MID1 protein for CAG repeat dependent translation, we performed MID1 knockdown before the dualluciferase reporter assay. While mRNA levels were unaffected by the knockdown of MID1 (Fig. 4a, for knockdown control see Fig.4c), MID1 knockdown led to a significant reduction of the reporter signal produced by the CAG47-repeat-construct (Fig. 4b). By contrast, neither the luciferase signal of the empty vector control nor of the construct with 14 CAGs were affected by the MID1 knockdown, suggesting a gain-of-function of the MID1-complex on mRNAs with pathologically expanded CAG repeat stretches. Alternative MID1-targeting siRNA oligonucleotides were used to confirm these findings (Supplementary Fig. S10).

Furthermore, we utilized a cell culture model for HD. HEKT-cell lines stably expressing HTT-exon 1 with either 20, 51 or 83 CAG repeats were transfected either with MID1- or non-silencing control siRNA oligonucleotides. Cells were analysed for HTT-exon 1 protein expression either by western blotting or, for aggregate formation, in a filter-aggregation assay, which was developed by Wanker et al ${ }^{33}$. While the knockdown of MID1 (knockdown control see Supplementary Fig. S11) did not influence the synthesis of HTT-exon 1 Q20 a
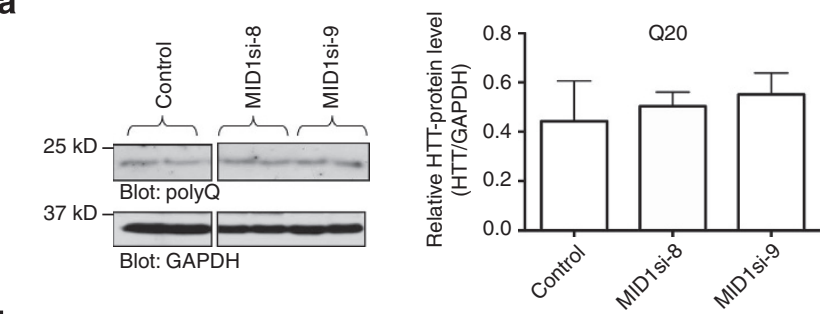

b

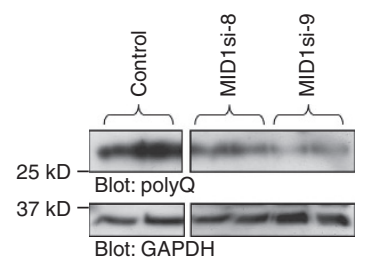

C

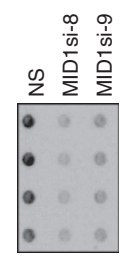

d
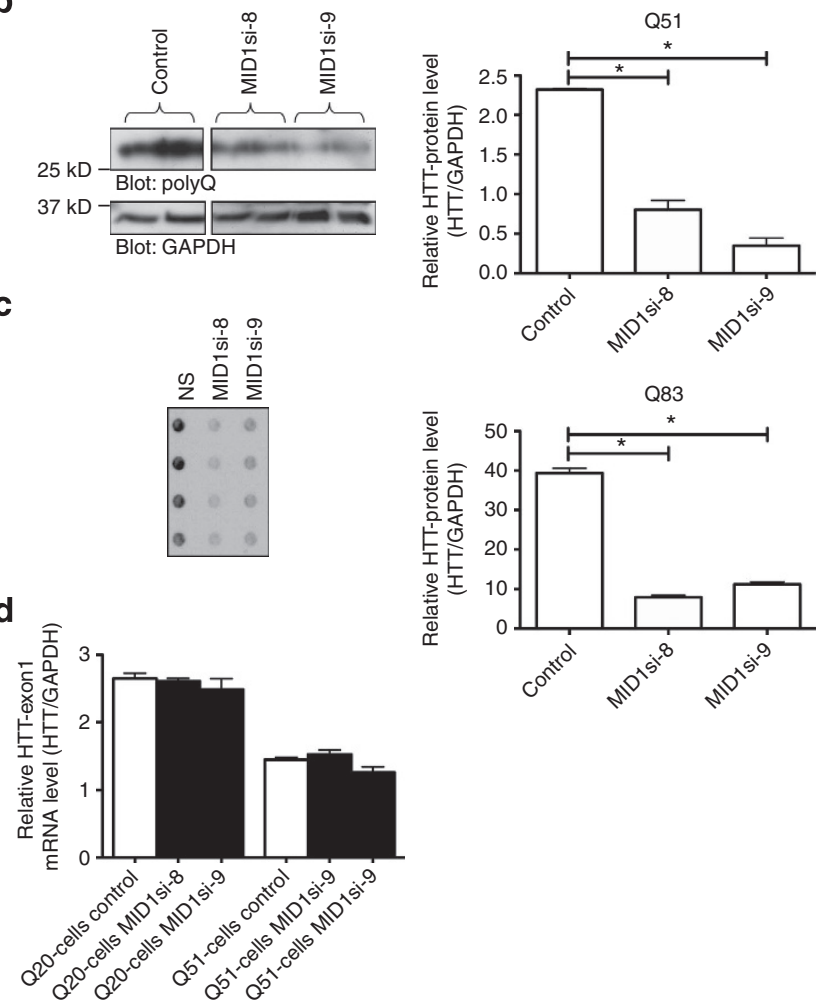

Figure 5 | MID1 regulates expression of aberrant HTT protein in a tissue culture model of HD. (a) Western blot analysis of cells stably expressing HTT-exon1 with 20 CAG repeats (Q20) after transfection with either nonsilencing control or MID1-specific siRNA oligonucleotides (MID1si-8, MID1si-9). Western blots showing HTT-exon1 (left), and quantifications of the bands (right) are shown. Samples were measured in triplicates. Mean values \pm s.d. are shown. $P<0.004$. (b) Western blot analysis of cells stably expressing HTT-exon1 with 51 CAG repeats (Q51) after transfection with either non-silencing control or MID1-specific siRNA oligonucleotides (MID1si-8, MID1si-9). Western blots showing HTT-exon1 (left), and quantifications of the bands (right) are shown. Samples were measured in triplicates. Mean values \pm s.d. are shown. $P<0.004$. (c) Filter-aggregation assay of HEKT-cells stably expressing HTT-exon1 with 83 CAG repeats (Q83) after transfection with either non-silencing control or MID1-specific siRNA oligonucleotides (MID1si-8, MID1si-9) is shown. HTT-aggregate detection (left), and quantifications (right) are shown. Samples were measured in triplicates. Mean values \pm s.d. are shown. $P<0.00002$. (d) Real-time PCR analysis of samples from $\mathbf{a}$ and $\mathbf{b}$ analysing HTT-exon1-mRNA expression normalized to glyceraldehyde 3-phosphate dehydrogenase (GAPDH). Samples were measured in quadruplicates. Mean values \pm s.d. are shown.

protein (Fig. 5a), the expression of HTT-exon 1 Q51 (Fig. 5b) and the formation of aggregates by HTT-exon 1 Q83 protein (Fig. 5c) decreased significantly after knockdown of MID1. RNA levels were not affected by MID1 knockdown (Fig. 5d). 
Translation of CAG repeat mRNAs is PP2A and mTOR dependent. MID1 is closely connected with PP2A and mTOR. In order to further confirm the interaction of MID1 and $\alpha 4$ with PP2Ac and S6K, an important target of the mTOR kinase, we performed co-immunoprecipitations with $\alpha 4$-V5 and MID1FLAG overexpressing HeLa cells. Both, PP2Ac and S6K clearly co-precipitated with $\alpha 4$ and MID1 (Supplementary Fig. S12). The interference between MID1, PP2A, mTOR and S6K made us ask the question if the effects of the MID1 protein on the translation efficiency of mRNAs with expanded CAG repeats are mediated by PP2A and mTOR activities.

MID1 is a negative regulator of PP2Ac levels and activity. If the effect of the MID1 knockdown on the translation efficiency of the HTT mRNA with expanded CAG repeats was mediated by the influence of MID1 on PP2A activity, inhibition of PP2A-for example, by treatment with its inhibitor $\mathrm{OA}-$ should be able to rescue the seen effect. For an easier handling of cells and treatments in the luciferase assay we established a HeLa cell line that stably expresses the firefly luciferase gene carrying 51 CAG repeats in its $3^{\prime}$-UTR. PP2A dependency of the MID1 knockdown effect was analysed in a blocking experiment where these cells were transfected either with a non-silencing or with a MID1specific siRNA oligonucleotide. In addition, cells were either mock treated or treated with OA, an inhibitor of PP2A. Results clearly show that the effect of MID1 knockdown on the synthesis of luciferase with 51 CAG repeats in its $3^{\prime}$-UTR can be completely blocked by OA treatment (Fig. 6a).

PP2A is a negative regulator of protein translation and opposes mTOR kinase activity on the translation regulators S6K and 4EBP1. Therefore, we asked if the translation of mRNAs with expanded CAG repeats is mTOR dependent. We performed the FRAP-based assay described above in the presence of either the mTOR inhibitor temsirolimus or the PP2A inhibitor OA. While OA significantly increased fluorescence recovery of HTT-exon1GFP Q49 (Fig. 6b, Supplementary Movie 4, Supplementary Fig. S9), temsirolimus decreased the fluorescence recovery clearly (Fig. 6c, Supplementary Movie 5). By contrast, OA treatment led to an only small increase of fluorescence recovery of HTT-exon1GFP Q17 and temsirolimus had no effect on the fluorescence recovery of this construct (Supplementary Fig. S13). The small OA effect can probably be explained by a residual MID1-binding ability of RNA with shorter CAG repeats, which we have seen in the RNA pull-down assays (Fig. 1).

In confirmation of the FRAP experiments, the cell line expressing firefly luciferase with 51 CAG repeats was treated with $\mathrm{OA}$ and temsirolimus. While OA stimulated luciferase production, temsirolimus almost entirely inhibited it (Fig. 6d). Together, these results demonstrate that the protein translation from mRNAs with expanded CAG repeats is an mTOR and PP2Adependent mechanism.

All these data indicate that the influence of the MID1-complex on the translation of HTT-exon 1 protein primarily affects mRNAs with CAG repeat expansions, but not with physiological repeat sizes and it involves $\mathrm{PP} 2 \mathrm{~A} / \mathrm{mTOR}$ activity-probably via regulation of the translation regulator $\mathrm{S} 6 \mathrm{~K}$.

\section{Discussion}

Expansion of CAG repeats is a common pathogenic mechanism in diseases like HD. Here we report a novel mechanism that regulates the translation of mRNAs with CAG repeat expansions. It involves MID1, PP2A and the PP2A/mTOR target S6K. Our data show that: (i) MID1, PP2Ac and S6K bind to CAG repeat stretches of the HTT mRNA in a repeat length dependent manner, (ii) protein synthesis increases with the size of the CAG repeat, (iii) knockdown of MID1 results in a significant reduction of protein a

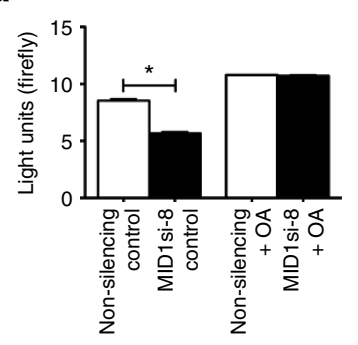

b

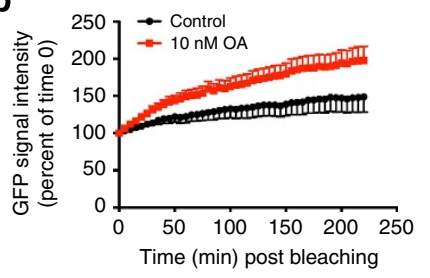

C

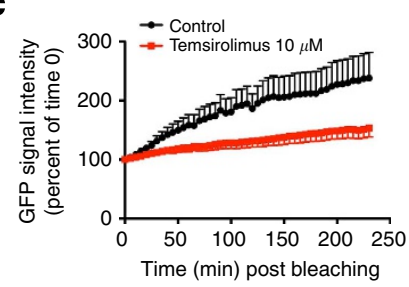

d

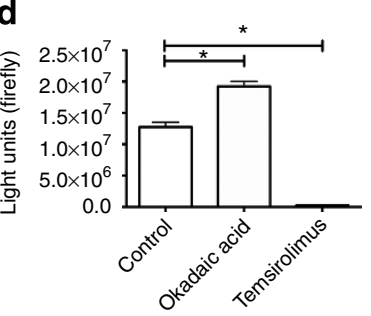

e

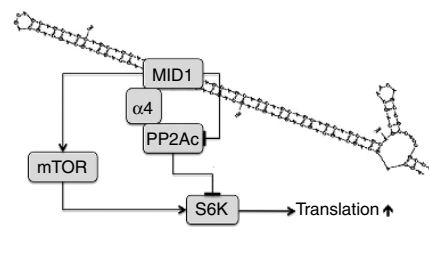

Figure 6 | Translation of mRNAs with expanded CAG repeats is PP2A and $\mathbf{m T O R}$ dependent. (a) Luciferase assay of a cell line stably expressing firefly luciferase carrying 51 CAG repeats in its $3^{\prime}$-UTR after transfection either with non-silencing control or MID1-specific siRNA oligonucleotides with or without okadaic acid (OA, $10 \mathrm{nM}, 12$ hours) treatment is shown. Samples were measured in triplicates. Mean values \pm s.d. are shown $(P<0.05)$. (b) FRAP-based assay showing translation rates of the GFPHTT-exon1 Q49 construct in HeLa cells treated with $(n=35)$ or without OA $(n=22)$. Data are presented as mean values \pm s.e.m. Curves showing signal recovery over time $(P<0.0001)$ are depicted. (c) FRAP-based assay as in $\mathbf{b}$ showing cells that were treated with $(n=38)$ or without temsirolimus $(n=29)(P<0.0001)$. Data are presented as mean values \pm s.e.m. (d) Luciferase assay of cells stably expressing firefly luciferase with 51 CAG repeats in its $3^{\prime}$-UTR. Cells were treated with or without either OA or temsirolimus. Samples were measured in triplicates. Mean values \pm s.d. are shown $(P<0.05)$. (e) Model: HTT mRNA with expanded CAG repeats recruits a protein complex containing MID1, $\alpha 4$, PP2AC and S6K. S6K induces protein translation and gets activated upon phosphorylation by mTOR. PP2A-as the mTOR opposing phosphatase-inactivates S6K by dephosphorylation. MID1 mediates binding of the protein complex to the CAG repeat mRNA and acts as a negative regulator of PP2A. MID1 also regulates $m$ TOR activity: in the absence of MID1 PP2Ac binds to its subunits $A$ and $B \alpha$ (instead of binding MID1 and $\alpha 4$ ) and then inhibits binding of mTOR to its interacting proteins for example, raptor, which leads to inhibition of mTOR in this protein complex (called TORC1) ${ }^{19}$. Therefore, increased binding of MID1 and S6K to HTT mRNA with elongated CAG repeats leads to an increased protein translation.

produced from mRNAs containing elongated CAG repeats, an effect that can be blocked by PP2A inhibitors and (iv) treatment with PP2A and mTOR inhibitors has opposing effects on the translation efficiency of mRNAs carrying expanded CAG repeats.

Until recently, solely the accumulation of polyQ-protein was believed to cause toxicity in polyQ expansion diseases like HD. Recent studies have shown that RNA-protein interactions of mutant poly-CAG RNAs might also contribute to toxicity: CAG 
repeat RNAs bind to and co-localize with the muscleblind (MBNL1) protein ${ }^{12}$. Originally MBNL1 had been found to bind to CUG or CCUG repeat mRNA-hairpin structures in patients with myotonic dystrophy (MD). In MD patients the sequestration of MBNL1 and other splicing factors by the mutant mRNAs leads to misregulated splicing, which is believed to be the underlying pathogenic mechanism in $\mathrm{MD}^{34-36}$. Similarly MBNL1 has been shown to bind CAG repeats ${ }^{37,38}$ leading to dysregulated alternative splicing of target mRNAs in CAG repeat expansion disorders $^{39}$. Furthermore, in a Drosophila model of MachadoJoseph disease, another CAG repeat expansion disorder, the MBNL1 protein enhanced CAG repeat RNA toxicity ${ }^{8,34-38}$. Thus, binding and sequestration of MBNL1 might be one possible mechanism contributing to RNA toxicity in CAG repeat expansion disorders (reviewed in Krzyzosiak et al. ${ }^{40}$ ). Here we show that expanded CAG repeat stretches in the HTT mRNA bind to the MID1 protein complex and that this protein complex controls this mRNAs' translation. Elongated CAG repeats in HD patients, therefore, probably induce translation of pathological protein in a gain-of-function like manner by recruiting the MID1 protein complex. Remarkably, siRNA mediated interruption of the MID1-protein complex particularly influenced translation of mRNAs with longer-for example, pathologicalCAG repeats and had no effect on mRNAs with normal repeat lengths. This indicates that by interfering with the MID1 protein complex it is possible to specifically influence protein translation from mRNAs carrying pathological sizes of repeats. These data make the MID1-complex a novel and very promising drug target for the development of a therapy for CAG repeat expansion disorders like HD aiming at the systematic reduction of pathological protein.

HD is caused by the expansion of a CAG repeat, which translates into an expanded polyQ tract. The expanded polyQ stretch leads to an increased cleavage of mutant HTT by caspases and calpains, and results in an accumulation of toxic N-terminal HTT-fragments, which form aggregates in $\mathrm{HD}$ brains ${ }^{41-45}$. These fragments get degraded via the ubiquitin-proteasome pathway and by autophagy. Reduction of proteasomal degradation has been found in cells carrying CAG repeat expansions ${ }^{46-49}$ and induction of both degradation pathways has been discussed as promising therapeutic strategies for CAG repeat expansion disorders including $\mathrm{HD}^{50-53}$

We show here that the MID1-PP2A complex controls the translation efficiencies of mRNAs that contain CAG repeat expansions. These findings suggest an RNA-based gain-offunction mechanism that leads to an overproduction of aberrant HTT protein in CAG repeat expansion carriers over time. The combination of both processes-a reduced clearance of mutant HTT protein plus an increase in translation of mutant HTT mRNA-could lead to a slow, over time accumulation of pathological protein in patients with HD. This would nicely explain (i) the slow accumulation of aggregates consisting of insoluble protein and (ii) the late onset of disease in CAG repeat expansion disorders. This hypothesis is further supported by data from Graham et al ${ }^{54}$. showing that in mice expressing varying levels of abnormal HTT protein increased protein levels account for a more severe phenotype, which is characterized by earlier onset, a more rapid progression and enhanced striatal volume loss.

We also show that the translation of mutant HTT mRNA depends on PP2A and mTOR activities. Our data suggest that induction of PP2A or inhibition of mTOR would result in a significant reduction of translation of mutant protein in HD patients and thereby would be able to reduce protein aggregation and perhaps delay the development of the disease phenotype. Interestingly, induction of autophagy, which has an important role in the clearance of aberrant protein in CAG repeat expansion disorders, has also been linked to mTOR inhibition ${ }^{52}$. mTOR inhibitors, therefore, have significant potential in therapies of CAG repeat expansion disorders because they combine a reduction of the synthesis of aberrant protein with an increase of its degradation.

Based on our observations we have developed a hypothetical working model: HTT mRNAs with pathologically expanded CAG repeats bind to the MID1 protein. MID1 recruits PP2Ac and the translation regulator S6K to these mRNAs. MID1 is a negative regulator of PP2Ac, thereby stimulating mTOR activity, the phosphorylation of $\mathrm{S} 6 \mathrm{~K}$, and protein translation ${ }^{19}$. This then would lead to an overproduction of aberrant protein coded by the mRNA and its expanded CAG repeat stretch (Fig. 6e).

\section{Methods}

RNA-structure prediction. Structure predictions of bp 146-415 of the HTTsequence NM_002111 with variable CAG repeat lengths were carried out using mfold (version 3.2) 55,56 .

RNA pull-down. HTT-CAG-repeat-motifs were PCR amplified, treated with proteinase $\mathrm{K}$ and purified by phenol-chloroform extraction. In vitro transcriptions were performed using the RiboMAX Large-scale RNA production system-T7 (Promega). The resulting biotinylated RNAs were purified by phenol-chloroform extraction. After preincubation of RNA with HeLa-protein extracts RNA pulldown was performed using streptavidin-coated Dynabeads (M-280, Dynal Biotech).

RNAse assisted chromatography. RNAse assisted chromatography was performed as described elsewhere ${ }^{27}$. In brief, HTT-CAG-repeat-motifs were PCR amplified, treated with proteinase $\mathrm{K}$ and purified by phenol-chloroform extraction. In vitro transcriptions were performed using the RiboMAX Large-scale RNA production system-T7 (Promega). The resulting RNAs were purified by phenolchloroform extraction. After incubation of RNA with HeLa-protein extracts RNAprotein complexes were washed, RNA-bound proteins were eluted using RNAse, and proteins were analysed on western blots.

Transient transfections and western blot. Autophagy positive and negative cells (Atg $+I+$ and Atg $-I-$, MEFs from mice deficient for Atg5) (kindly provided by Dr Noboru Mizushima ${ }^{29}$ ) were transfected with pEGFP-C1 and HTT-500AAQ17/49, resuspended in Magic-Mix, and western blotted on polyvinylidene difluoride membranes (Roche). For quantification of western blots each sample was analysed in triplicates, band intensities were measured using Imagequant5.2. Data shown represent mean \pm s.d. Statistical significances were evaluated using $t$-test (two-tailed, homoscedastic).

Expression constructs. Expression-vectors were transfected using Lipofectamine 2000 (Invitrogen). Expression-vector constructs are listed (Supplementary Table S1).

In vitro translation. Luciferase with CAG-repeat-motifs in the $3^{\prime}$-UTR was PCR amplified and subjected to in vitro transcription using the MMESSAGE mMACHINE Kit (Ambion). In vitro transcribed RNA was phenol-chloroform purified and analysed by means of in vitro translation using the Flexi Rabbit Reticulocyte Lysate System (Promega).

Luciferase reporter assays. U373MG/HeLa cells co-transfected with reporter plasmids (pGL3-vectors) and renilla luciferase (pRL) or a stable cell line expressing HTT-exon1 CAG 51 in the $3^{\prime}$-UTR of firefly luciferase (HeLa, TRex-system, Invitrogen) were subjected to luciferase reporter assays. Reporter assays were carried out using the Dual-Luciferase Reporter 1000 Assay System (Promega). Samples were analysed in quadruplicates. Data shown represent mean \pm s.d. Statistical significances were evaluated using $t$-test (two-tailed, homoscedastic).

Real-time PCR. Total RNA was isolated using the RNeasy Mini Kit (Qiagen). cDNA was synthesized using the TaqMan reverse transcription reagents kit (Applied Biosystems) and real-time PCR was carried out using the SYBRGreen PCR master mix (Applied Biosystems). Samples were analysed in quadruplicates. Data shown represent mean \pm s.d. Statistical significances were evaluated using $t$-test (two-tailed, homoscedastic).

Primers. Primers are listed in Supplementary Table S2. 
Antibodies. Antibodies are listed in Supplementary Table S3.

Short interfering RNAs. siRNAs were transfected using Oligofectamine (Invitrogen) or Polyfect (Qiagen). Sequences of siRNAs are listed in Supplementary Table S4.

Filter retardation assay. HEKT-cells stably expressing HTT-exon 1 with 83 CAG repeats ${ }^{3}$ were transfected with siRNAs. Filter retardation assays were performed as described elsewhere ${ }^{3}$. Samples were analysed in quadruplicates. Data shown represent mean \pm s.d. Statistical significances were evaluated using $t$-test (twotailed, homoscedastic)

HD-mice. Wild-type or HD knock-in mice carrying 111 CAG repeats in the endogenous HTT-gene (B6.129-Htt ${ }^{\text {tm5Mem}} / \mathrm{J}$, Jackson's Lab stock No.003598) were decapitated and brains were rapidly dissected in nitrogen. Protein extracts were dissolved in Magic-Mix (48\% urea, $15 \mathrm{mM}$ Tris-HCL pH 7.5, 8.7\% glycerine, $1 \%$ SDS, $0,004 \%$ Bromophenolblue, $143 \mathrm{mM}$ Mercaptoethanol), separated on 6\% SDS gels and analysed on western blots. Bands on the western blots were analysed in triplicates and quantified using Aida Image Analyser Software v.3.28. Data shown represent mean \pm s.d. Statistical significances were evaluated using $t$-test (twotailed, homoscedastic)

FRAP-based assays. HeLa cells were transfected with constructs expressing HTTexon1 fused to GFP. Twelve to twenty hours after transfection cells were analysed using a Zeiss LSM710NLO and a Zeiss LSM700 with a X20 objective. The GFPsignal of individual cells was bleached with a 488 argon laser of high intensity. Fluorescence signal was imaged over $4 \mathrm{~h}$ using reduced laser power to minimize photobleaching. Fluorescence signal was measured as the sum of the pixel over the cell area in each picture. The resulting total cell fluorescence was normalized for the whole time series to the second frame after bleaching, which was set to $100 \%$. Bleaching experiments were performed on 14-38 cells for each experimental condition. Finally, mean values and s.e. values were calculated from normalized fluorescence recovery curves of single cells. To estimate statistical differences between different experimental conditions, normalized recovery curves of single cells were integrated over time $(0-220 \mathrm{~min})$ and rank-sum test was applied to distributions of resulting 'area under the curve' values.

Co-immunoprecipitation. For co-immunoprecipitation experiments, HeLa cells were transfected with MID1-FLAG and $\alpha 4$-V5. Forty-eight hours after transfection cells were lysed by sonication in IP-buffer (50 mM Tris pH 7.5, $2.5 \mathrm{mM} \mathrm{MgCl}_{2}$, $100 \mathrm{mM} \mathrm{NaCl}, 1 \mathrm{mM}$ dithiothreitol, complete (protease inhibitor cocktail; Roche)) either with or without addition of RNAse (NEB). Immunoprecipitation was carried out using FLAG- or V5-specific antibodies or unspecific mouse IgG as negative controls in combination with protein A-/ or G-agarose (Roche) following the manufacturer's instructions and immunoprecipitates were analysed on western blots.

Statistical analysis. Statistical analyses were performed using GraphPad Prism 5 software. Student's $t$-test was used for two-group comparisons. For multiple comparisons data were analysed by one-way analysis of variance with post hoc Dunnett's or Bonferonni test, as appropriate. For FRAP based assays, rank-sum test was applied to distributions of resulting 'area under the curve' values.

\section{References}

1. Gil, J. M. \& Rego, A. C. Mechanisms of neurodegeneration in Huntington's disease. Eur. J. Neurosci. 27, 2803-2820 (2008).

2. DiFiglia, M. et al. Aggregation of huntingtin in neuronal intranuclear inclusions and dystrophic neurites in brain. Science 277, 1990-1993 (1997).

3. Scherzinger, E. et al. Huntingtin-encoded polyglutamine expansions form amyloid-like protein aggregates in vitro and in vivo. Cell 90, 549-558 (1997)

4. Everett, C. M. \& Wood, N. W. Trinucleotide repeats and neurodegenerative disease. Brain 127, 2385-2405 (2004)

5. Rubinsztein, D. C. How does the Huntington's disease mutation damage cells? Sci. Aging Knowledge Environ. PE26 (2003).

6. Paulson, H. L. et al. Intranuclear inclusions of expanded polyglutamine protein in spinocerebellar ataxia type 3. Neuron 19, 333-344 (1997).

7. Taylor, J. P., Hardy, J. \& Fischbeck, K. H. Toxic proteins in neurodegenerative disease. Science 296, 1991-1995 (2002).

8. Li, L. B., Yu, Z., Teng, X. \& Bonini, N. M. RNA toxicity is a component of ataxin-3 degeneration in Drosophila. Nature 453, 1107-1111 (2008).

9. Sobczak, K. \& Krzyzosiak, W. J. CAG repeats containing CAA interruptions form branched hairpin structures in spinocerebellar ataxia type 2 transcripts. J. Biol. Chem. 280, 3898-3910 (2005).

10. Sobczak, K., de Mezer, M., Michlewski, G., Krol, J. \& Krzyzosiak, W. J. RNA structure of trinucleotide repeats associated with human neurological diseases. Nucleic Acids Res. 31, 5469-5482 (2003).
11. Kiliszek, A., Kierzek, R., Krzyzosiak, W. J. \& Rypniewski, W. Atomic resolution structure of CAG RNA repeats: structural insights and implications for the trinucleotide repeat expansion diseases. Nucleic Acids Res. 38, 8370-8376 (2010).

12. de Mezer, M., Wojciechowska, M., Napierala, M., Sobczak, K. \& Krzyzosiak, W. J. Mutant CAG repeats of Huntingtin transcript fold into hairpins, form nuclear foci and are targets for RNA interference. Nucleic Acids Res. 39, 3852-3863 (2011).

13. McLaughlin, B. A., Spencer, C. \& Eberwine, J. CAG trinucleotide RNA repeats interact with RNA-binding proteins. Am. J. Hum. Genet. 59, 561-569 (1996).

14. Ranum, L. P. \& Cooper, T. A. RNA-mediated neuromuscular disorders. Annu Rev. Neurosci. 29, 259-277 (2006).

15. Hieronymus, H. \& Silver, P. A. A systems view of mRNP biology. Genes Dev 18, 2845-2860 (2004).

16. Aranda-Orgilles, B. et al. The PP2a-specific ubiquitin ligase Mid1 is a sequence-dependent regulator of translation efficiency controlling 3 phosphoinositide dependent protein kinase-1 (PDPK-1). J. Biol. Chem. 286, 39945-39957 (2011).

17. Aranda-Orgilles, B. et al. The opitz syndrome gene product MID1 assembles a microtubule-associated ribonucleoprotein complex. Hum. Genet. 123, 163-176 (2008).

18. Trockenbacher, A. et al. MID1, mutated in Opitz syndrome, encodes an ubiquitin ligase that targets phosphatase 2A for degradation. Nat. Genet. 29, 287-294 (2001).

19. Liu, E., Knutzen, C. A., Krauss, S., Schweiger, S. \& Chiang, G. G. Control of mTORC1 signaling by the Opitz syndrome protein MID1. Proc. Natl Acad. Sci. USA 108, 8680-8685 (2011).

20. Kim, D. H. et al. mTOR interacts with raptor to form a nutrient-sensitive complex that signals to the cell growth machinery. Cell 110, 163-175 (2002).

21. Holz, M. K., Ballif, B. A., Gygi, S. P. \& Blenis, J. mTOR and S6K1 mediate assembly of the translation preinitiation complex through dynamic protein interchange and ordered phosphorylation events. Cell 123, 569-580 (2005).

22. Jackson, R. J. \& Wickens, M. Translational controls impinging on the 5'untranslated region and initiation factor proteins. Curr. Opin. Genet. Dev. 7, 233-241 (1997).

23. Nielsen, F. C., Ostergaard, L., Nielsen, J. \& Christiansen, J. Growth-dependent translation of IGF-II mRNA by a rapamycin-sensitive pathway. Nature 377, 358-362 (1995).

24. Gebauer, F. \& Hentze, M. W. Molecular mechanisms of translational control. Nat. Rev. Mol. Cell. Biol. 5, 827-835 (2004).

25. Ma, X. M. \& Blenis, J. Molecular mechanisms of mTOR-mediated translational control. Nat. Rev. Mol. Cell. Biol. 10, 307-318 (2009).

26. Orgillés, B. A. Inaugural Dissertation (Freie Universität Berlin, 2006).

27. Michlewski, G. \& Caceres, J. F. RNase-assisted RNA chromatography. RNA 16, 1673-1678 (2010).

28. Sarkar, S., Ravikumar, B., Floto, R. A. \& Rubinsztein, D. C. Rapamycin and mTOR-independent autophagy inducers ameliorate toxicity of polyglutamineexpanded huntingtin and related proteinopathies. Cell Death Differ. 16, 46-56 (2008).

29. Kuma, A. et al. The role of autophagy during the early neonatal starvation period. Nature 432, 1032-1036 (2004).

30. Sobczak, K. \& Krzyzosiak, W. J. Imperfect CAG repeats form diverse structures in SCA1 transcripts. J. Biol. Chem. 279, 41563-41572 (2004).

31. Dixon, K. T., Cearley, J. A., Hunter, J. M. \& Detloff, P. J. Mouse Huntington's disease homolog mRNA levels: variation and allele effects. Gene Expr. 11, 221-231 (2004).

32. Dragatsis, I. et al. CAG repeat lengths $>$ or $=335$ attenuate the phenotype in the R6/2 Huntington's disease transgenic mouse. Neurobiol. Dis. 33, 315-330 (2009).

33. Wanker, E. E. et al. Membrane filter assay for detection of amyloid-like polyglutamine-containing protein aggregates. Methods Enzymol. 309, 375-386 (1999).

34. Ho, T. H. et al. Muscleblind proteins regulate alternative splicing. EMBO J. 23 , 3103-3112 (2004).

35. Kanadia, R. N. et al. A muscleblind knockout model for myotonic dystrophy. Science 302, 1978-1980 (2003).

36. Osborne, R. J. \& Thornton, C. A. RNA-dominant diseases. Hum. Mol. Genet. 15 (Spec No 2) R162-R169 (2006).

37. Yuan, Y. et al. Muscleblind-like 1 interacts with RNA hairpins in splicing target and pathogenic RNAs. Nucleic Acids Res. 35, 5474-5486 (2007).

38. Ho, T. H. et al. Colocalization of muscleblind with RNA foci is separable from mis-regulation of alternative splicing in myotonic dystrophy. J. Cell Sci. 118, 2923-2933 (2005).

39. Mykowska, A., Sobczak, K., Wojciechowska, M., Kozlowski, P. \& Krzyzosiak, W. J. CAG repeats mimic CUG repeats in the misregulation of alternative splicing. Nucleic Acids Res. 39, 8938-8951 (2011).

40. Krzyzosiak, W. J. et al. Triplet repeat RNA structure and its role as pathogenic agent and therapeutic target. Nucleic Acids Res. 40, 11-26 (2012). 
41. Qin, Z. H. \& Gu, Z. L. Huntingtin processing in pathogenesis of Huntington disease. Acta. Pharmacol. Sin. 25, 1243-1249 (2004).

42. Landles, C. et al. Proteolysis of mutant huntingtin produces an exon 1 fragment that accumulates as an aggregated protein in neuronal nuclei in Huntington disease. J. Biol. Chem. 285, 8808-8823 (2010).

43. Ratovitski, T. et al. Mutant huntingtin N-terminal fragments of specific size mediate aggregation and toxicity in neuronal cells. J. Biol. Chem. 284, 10855-10867 (2009).

44. Majumder, P., Raychaudhuri, S., Chattopadhyay, B. \& Bhattacharyya, N. P. Increased caspase-2, calpain activations and decreased mitochondrial complex II activity in cells expressing exogenous huntingtin exon 1 containing CAG repeat in the pathogenic range. Cell. Mol. Neurobiol. 27, 1127-1145 (2007)

45. Southwell, A. L. et al. Perturbation with intrabodies reveals that calpain cleavage is required for degradation of huntingtin exon 1. PLOS ONE 6, e16676 (2011).

46. Wyttenbach, A. et al. Effects of heat shock, heat shock protein 40 (HDJ-2), and proteasome inhibition on protein aggregation in cellular models of Huntington's disease. Proc. Natl Acad. Sci. USA 97, 2898-2903 (2000).

47. Jana, N. R., Zemskov, E. A., Wang, G. \& Nukina, N. Altered proteasomal function due to the expression of polyglutamine-expanded truncated $\mathrm{N}$-terminal huntingtin induces apoptosis by caspase activation through mitochondrial cytochrome c release. Hum. Mol. Genet. 10, 1049-1059 (2001).

48. Bence, N. F., Sampat, R. M. \& Kopito, R. R. Impairment of the ubiquitinproteasome system by protein aggregation. Science 292, 1552-1555 (2001)

49. Bennett, E. J. et al. Global changes to the ubiquitin system in Huntington's disease. Nature 448, 704-708 (2007)

50. Li, X. et al. Inhibiting the ubiquitin-proteasome system leads to preferential accumulation of toxic N-terminal mutant huntingtin fragments. Hum. Mol. Genet. 19, 2445-2455 (2010).

51. Roscic, A., Baldo, B., Crochemore, C., Marcellin, D.0 \& Paganetti, P. Induction of autophagy with catalytic mTOR inhibitors reduces huntingtin aggregates in a neuronal cell model. J. Neurochem. 119, 398-407 (2011).

52. Ravikumar, B. et al. Inhibition of mTOR induces autophagy and reduces toxicity of polyglutamine expansions in fly and mouse models of Huntington disease. Nat. Genet. 36, 585-595 (2004).

53. Tsvetkov, A. S. et al. A small-molecule scaffold induces autophagy in primary neurons and protects against toxicity in a Huntington disease model. Proc. Natl Acad. Sci. USA 107, 16982-16987 (2010).
54. Graham, R. K. et al. Levels of mutant huntingtin influence the phenotypic severity of Huntington disease in YAC128 mouse models. Neurobiol. Dis. 21, 444-455 (2006).

55. Mathews, D. H., Sabina, J., Zuker, M. \& Turner, D. H. Expanded sequence dependence of thermodynamic parameters improves prediction of RNA secondary structure. J. Mol. Biol. 288, 911-940 (1999).

56. Zuker, M. Mfold web server for nucleic acid folding and hybridization prediction. Nucleic Acids Res. 31, 3406-3415 (2003).

\section{Acknowledgements}

We thank Thomas Schöndorf for technical assistance, Ireen König, Christoph Moehl and Philipp Denner for sharing expertise in microscopy and FRAP data analysis, and Ina Vorberg and Daniele Bano for careful review of this manuscript. This work was supported by the Volkswagen Stiftung (Lichtenberg fellowship to SS), AtaxiaUK, Tenovus Scotland, the Medical University Innsbruck (IFTZ-03), the Austrian Science Fund (FWFSFB021-Project 03 and TRP233) and the Tiroler Zukunftsstiftung.

\section{Author contributions}

S.K., N.G., S.D., C.C., E.J., D.R. and C.A. performed experiments, S.K., R.S. and S.S. planned and designed experiments, S.K. and N.G. analysed the data, S.K. and S.S. wrote the paper. E.W. and M.L. provided constructs, developed analytical tools; S.B. and R.S. gave critical advice. All authors discussed the results and commented on the manuscript.

\section{Additional information}

Supplementary Information accompanies this paper at http://www.nature.com/ naturecommunications

Competing financial interests: The authors declare no competing financial interests.

Reprints and permission information is available online at http://npg.nature.com/ reprintsandpermissions/

How to cite this article: Krauß, S. et al. Translation of HTT mRNA with expanded CAG repeats is regulated by the MID1-PP2A protein complex. Nat. Commun. 4:1511 doi: 10.1038/ncomms2514 (2013). 